\title{
A PROPERTY OF REGULAR MEASURES IN LOCALLY COMPACT HAUSDORFF SPACES ${ }^{1}$
}

\author{
R. P. PAKSHIRAJAN
}

1. Introduction. Let $G$ denote a locally compact Hausdorff topological space. $\Theta$ is the class of Borel sets of $G$, i.e., the $\sigma$-ring generated by the class $\mathcal{C}$ of compact subsets of $G$. Let $D$ be the class of sets whose intersection with every compact set is a Borel set. Note that $D$ is a $\sigma$-field containing the open sets of $G$. Given a measure $\mu$ (a non-negative countably additive set function not identically equal to zero) on $B[D]$, denote by $B_{\mu}\left[D_{\mu}\right]$ the class of sets which either belong to $\leftrightarrow[D]$ or which differ from a member of $B[D]$ by a subset of a set of $\mu$-measure zero.

A measure $\mu$ defined on $\&[D]$ is said to be regular if (i) $\mu(C)<\infty$ for every $C \in \mathcal{C}$ and (ii) for every $B \in \mathbb{B}[\mathbb{D}], \mu(B)=\sup \{\mu(C): C \in \mathfrak{C}, C \subseteq B\}$. Given a regular measure $\mu$ on $B$ extend it to $D$ by defining $\mu(D)$ $=\sup \{\mu(D \cap C): C$ compact $\}$ for all $D \in D$. Observe that $\mu$ so extended to $D$ is regular. Below we shall assume that either $\mu$ on $D$ is given or that $\mu$ on $B$ is extended to $D$ as above.

The object of this paper is to prove the following

THEOREM. To every regular measure $\mu$ on $D$ there corresponds a unique closed set $A_{\mu}$, the carrier of $\mu$, with $\mu\left(G \sim A_{\mu}\right)=0$ and $\mu(U)>0$ for every non-null relatively open subset $U$ of $A_{\mu}$.

This theorem when $G$ is compact is due to Wendel [1]. His proof is direct using heavily compactness of $G$.

2. Proof of Theorem. As $\mu \neq \equiv 0$ and as $\mu$ is regular we can find a compact set $C$ with $\mu(C)>0$. As the space is locally compact and as $C$ is a compact subset we can, by considering the covering of $C$ by compact neighbourhoods of the points of $C$, find an open set $A \supseteq C$ with $\bar{A}$ compact. It is clear that $\mu(A)>0$ since $A \supseteq C$. A slight modification of the proof of [1, Lemma 3$]$ then yields a compact set $A_{1} \subseteq \bar{A}$ such that every non-null relatively open subset of $A_{1}$ has positive $\mu$-measure and $\mu\left(\bar{A} \sim A_{1}\right)=0$. Write $B=A \cap A_{1}$. As $A \in B$ and

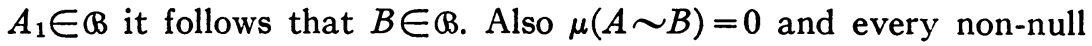
relatively open subset of $B$ has positive $\mu$-measure.

Received by the editors June 30, 1960.

1 This paper contains a part of the author's doctoral dissertation written under the direction of Professor Herman Rubin at the University of Oregon, and was supported by an Office of Ordnance Research Grant. 
Consider now the collection of all open subsets $A$ which admit of a subset $B \in D_{\mu}$ whose non-null relatively open subsets have positive measure and $\mu(A \sim B)=0$. Let $\mathcal{E}$ denote the family of all such pairs $(A, B)$. In view of earlier remarks this class is nonempty. Partially order this class by: $\left(A_{1}, B_{1}\right) \leqq\left(A_{2}, B_{2}\right)$ if and only if $A_{1} \subseteq A_{2}$ and $B_{1}=A_{1} \cap B_{2}$.

We will show that every chain in $\varepsilon$ has an upper bound. It would then follow that $\mathcal{E}$ has a maximal element.

Let $\left\{\left(A_{\alpha}, B_{\alpha}\right), \alpha \in \Sigma\right\}$ be a chain from $\varepsilon$. Write $A=\bigcup_{\alpha \in \Sigma} A_{\alpha}$; $B=\mathrm{U}_{\alpha \in \Sigma} B_{\alpha} . A$ is thus an open set and hence belongs to $D$. We will show that $B \in D_{\mu}$ and that non-null relatively open subsets of $B$ have positive measure.

Let $\mu^{*}, \mu_{*}$ be respectively the outer and inner measures induced by $\mu$ and let $K$ be any compact subset. Suppose that $C \subseteq A \cap K, C$ a compact subset. Hence $C \subseteq A$ and $\left\{A_{\alpha}, \alpha \in \Sigma\right\}$ is an open covering for the compact set $C$. Therefore there exists a finite subcovering from this. As the $A_{\alpha}$ 's are linearly ordered, it follows that $C \subseteq A_{\theta}$ for some $\theta \in \Sigma$. Now, $\mu(C)=\mu\left(C \cap A_{\theta}\right)=\mu\left(C \cap B_{\theta}\right)$ since $\mu\left(A_{\theta} \sim B_{\theta}\right)=0$. Write $C_{1}=C \cap B_{\theta}$. Hence $C_{1}$ is a Borel set, $\mu(C)=\mu\left(C_{1}\right)$ and $C_{1} \subseteq B \cap K$. Regularity of $\mu$ now gives $\mu_{*}(B \cap K) \geqq \mu(A \cap K)$. That $\mu^{*}(B \cap K)$ $\leqq \mu(A \cap K)$ follows from the fact that $B \cap K \subseteq A \cap K$. Hence

$$
\mu^{*}(B \cap K)=\mu_{*}(B \cap K)=\mu(A \cap K) .
$$

This implies that $B \cap K \in \mathbb{B}_{\mu}$. As the compact set $K$ is arbitrary, $B \in D_{\mu}$. Also $\mu(B \cap K)=\mu(A \cap K)$. As $\mu$ is regular, we have $\mu(A \sim B)$ $=0$.

Let $V$ be any open set with $B \cap V$ non-null. Hence there is a $B_{\alpha}$, $\alpha \in \Sigma$ such that $B_{\alpha} \cap V$ is non-null. Therefore

$$
\mu(B \cap V) \geqq \mu\left(B_{\alpha} \cap V\right)>0 .
$$

Thus $(A, B) \in \mathcal{E}$. That $\left(A_{\alpha}, B_{\alpha}\right) \leqq(A, B)$ for all $\alpha \in \Sigma$ is evident. We have therefore proved that every chain from $\varepsilon$ has an upper bound. This implies that $\mathcal{E}$ has a maximal element. Let this be $(M, N)$.

We claim $M=G$. For, if not, two cases can arise.

Case (i) $\mu(G \sim M)=0$. In this case we see immediately that $(G, N)$ $\in \varepsilon$. Further $(M, N) \leqq(G, N)$ and the two elements are not the same, thus contradicting the maximality of $(M, N)$.

Case (ii) $\mu(G \sim M)>0$. By the regularity of $\mu$, there exists therefore a compact set $K \subseteq(G \sim M)$ with $\mu(K)>0$. As explained at the beginning of the proof, we can find an open set $U \supseteq K$ such that $\bar{U}$ is compact. From all this we conclude that there exists a point $x \in(G \sim M)$ 
and an open set $U$ such that $x \in U, \bar{U}$ is compact and $\mu(U)>0$. We can then find a Borel set $V$ such that $(U, V) \in \mathcal{E}$. Let $M_{1}=M \cup U$ and $N_{1}=N \cup V_{1}$ where $V_{1}=(V \sim M)$. From the fact that non-null relatively open subsets of $N$ and of $V$ have positive measures we see that $N_{1}$ has this property too. Notice also that $M_{1}$ is open, $N_{1} \subseteq M_{1}$ and $\mu\left(M_{1} \sim N_{1}\right)=0$. Thus $\left(M_{1}, N_{1}\right) \in \varepsilon$. Obviously however $M \subset M_{1}$ and $N=M \cap N_{1}$. So $(M, N) \leqq\left(M_{1}, N_{1}\right)$ and these two elements of $\varepsilon$ are not the same. This contradicts the maximality of $(M, N)$. Therefore $G=M$, as we claimed.

Let $A_{\mu}=\bar{N}$. Then $\mu\left(G \sim A_{\mu}\right)=0$.

Every open set having a non-null intersection with $\bar{N}$ has a nonnull intersection with $N$. Therefore every non-null relatively open subset of $A_{\mu}$ has positive measure.

To prove the uniqueness of $A_{\mu}$, assume, if possible, a second closed set $A \neq A_{\mu}$ such that $\mu(G \sim A)=0$ and such that every non-null relatively open subset of $A$ has positive $\mu$-measure. If $A \nsubseteq A_{\mu}$, then $A \sim A_{\mu}$ is a non-null relatively open subset of $A$. Therefore $0<\mu\left(A \sim A_{\mu}\right)$ $\leqq \mu\left(G \sim A_{\mu}\right)=0$, a contradiction. Hence $A \subseteq A_{\mu}$. Similarly $A_{\mu} \subseteq A$. Thus $A=A_{\mu}$ and the proof is complete.

\section{REFERENCE}

1. J. G. Wendel, Haar measure and the semi-group of measures on a compact group, Proc. Amer. Math. Soc. vol. 5 (1954) pp. 923-929.

UNIVERSITY OF OREgON AND

OSMANIA UNIVERSITY, INDIA 